\title{
TW Hydrae: multi-wavelength interferometry of a transition disk
}

\author{
J. Menu ${ }^{1,2} \dagger$, R. van Boekel ${ }^{2}$, T. Henning ${ }^{2}$, M. Benisty ${ }^{3}$, \\ C. J. Chandler ${ }^{4}$, H. Linz ${ }^{2}$, C. Waelkens ${ }^{1}$, S. M. Andrews ${ }^{5}$, N. Calvet ${ }^{6}$, \\ J. M. Carpenter ${ }^{7}$, S. A. Corder ${ }^{8}$, A. T. Deller ${ }^{9}$, C. P. Dullemond ${ }^{10}$,
} J. S. Greaves ${ }^{11}$, R. J. Harris ${ }^{5}$, A. Isella ${ }^{7}$, W. Kwon ${ }^{12}$, J. Lazio ${ }^{13}$, L. G. Mundy ${ }^{14}$, L. M. Perez ${ }^{7}$, L. Ricci ${ }^{7}$, A. I. Sargent ${ }^{7}$, S. Storm ${ }^{14}$, L. Testi ${ }^{15,16}$ \& D. J. Wilner ${ }^{5}$

${ }^{1}$ Instituut voor Sterrenkunde, KU Leuven, Belgium

${ }^{2}$ Max Planck Institut für Astronomie, Heidelberg, Germany

${ }^{3}$ Institut de Planétologie et d'Astrophysique de Grenoble, France

${ }^{4}$ National Radio Astronomy Observatory, Socorro, USA

${ }^{5}$ Harvard-Smithsonian Center for Astrophysics, Cambridge, USA

${ }^{6}$ Department of Astronomy, University of Michigan, Ann Arbor, USA

${ }^{7}$ Astronomy Department, California Institute of Technology, Pasadena, USA

${ }^{8}$ Joint ALMA Observatory, Santiago, Chile

${ }^{9}$ ASTRON, The Netherlands Institute for Radio Astronomy, Dwingeloo, The Netherlands

${ }^{10}$ Institute for Theoretical Astrophysics, University of Heidelberg, Germany

${ }^{11}$ School of Physics and Astronomy, University of St. Andrews, UK

${ }^{12}$ Department of Astronomy, University of Illinois, Urbana, USA

${ }^{13}$ Jet Propulsion Laboratory, California Institute of Technology, Pasadena, USA

${ }^{14}$ Department of Astronomy, University of Maryland, College Park, USA

${ }^{15}$ European Southern Observatory, Garching, Germany

${ }^{16}$ INAF-Osservatorio Astrofisico di Arcetri, Firenze, Italy

\begin{abstract}
For over a decade, the structure of the inner "hole" in the transition disk around TW Hydrae has been a subject of debate. To probe the innermost regions of the protoplanetary disk, observations at the highest possible spatial resolution are required. We present new interferometric data of TW Hya from near-infrared to millimeter wavelengths. We confront existing models of the disk structure with the complete data set and develop a new, detailed radiativetransfer model. This model is characterized by: 1) a spatial separation of the largest grains from the small disk grains; and 2) a smooth inner rim structure, rather than a sharp disk edge.
\end{abstract}

Keywords. stars: individual (TW Hya), planetary systems: protoplanetary disks, techniques: interferometric

\section{Introduction}

The potential discovery of (proto)planetary bodies inside gaps of transition disks (e.g. Quanz et al. 2013) provides the ultimate justification for calling circumstellar disks around young stars "protoplanetary". Conversely, it also suggests that a transition-disk phase might be fundamental in the formation of planetary systems.

A well-known example of a protoplanetary disk with an inner hole is TW Hydrae (TW Hya). Being the closest accreting $\mathrm{T}$ Tauri star, it has been the target of many studies on the geometry of the circumstellar dust distribution. Calvet et al. (2002) were the first to conclude that the low near-infrared emission from the disk indicates a lack of dust close to the star. Their proposed model had a gap radius of $4 \mathrm{AU}$, with only a very low amount

$\dagger \mathrm{PhD}$ fellow of the Research Foundation - Flanders; email: jonathanm@ster.kuleuven.be 
of dust inside the central hole. Whereas millimeter observations seemed to confirm this geometry (Hughes et al. 2007), a model with a much smaller central hole $(\sim 0.7 \mathrm{AU}$ in radius) was used to explain mid-infrared interferometry (Ratzka et al. 2007). Later models proposed hybrid structures with an additional dust ring in the hole (Akeson et al. 2011) or even a self-luminous companion (Arnold et al. 2012).

Clearly, the disk of TW Hya turns out to be more complex than initial models suggested, and a final consensus on what currently is the "best" model is far from being reached. Understanding the disk structure is essential for answering at least 2 questions:

- What are the dominant processes that determine the disk dispersal? The inner-disk structure might bear the signposts of planet formation.

- What is the gas structure of the disk? The dust distribution can be interpreted as a "boundary condition" for studying the gas dynamics/chemistry.

The goal of this contribution is not to answer the above questions, but to see how well we currently understand the structure of the TW Hya disk. We collected high angular resolution data from near-infrared to millimeter wavelengths (Sect. 2), and give the results of comparing the data with published models in Sect. 3. Finally, in Sect. 4 we describe the new model we propose for the geometry of the TW Hya disk.

\section{Interferometric data of TW Hya}

Below, we introduce the new and archival interferometric data collected for this study. We also collected the spectral energy distribution (SED) from UV to mm-wavelengths.

Near-infrared interferometry (VLTI/PIONIER). Data was obtained with the PIONIER instrument on the Very Large Telescope Interferometer (VLTI) in 2011. The H-band observations $(\lambda=1.65 \mu \mathrm{m})$ for baselines up to $130 \mathrm{~m}$ have an average visibility of $V^{2}=0.968 \pm 0.005$, indicating that TW Hya is largely unresolved. The $1.6 \%$ of missing correlated flux to reach $V^{2}=1$ can be interpreted as evidence for an overresolved scattering component from the outer disk.

Mid-infrared interferometry (VLTI/MIDI). N-band $(\lambda=8-13 \mu \mathrm{m})$ observations of different epochs indicate a characteristic scale of $R=0.7 \mathrm{AU}$ for the mid-infrared emission. The data indicates no significant inclination for the inner disk region. We combine the observations into a 25-m baseline and a 50-m baseline correlated flux curve.

Sub-mm interferometry (SMA). Our analysis includes the sub-mm interferometry presented in Andrews et al. (2012), obtained using the Submillimeter Array (SMA; $\lambda=$ $870 \mu \mathrm{m})$. The data is deprojected as in the original paper.

Millimeter interferometry (VLA). The final piece of information is a new 9-mm data set obtained with the Karl G. Jansky Very Large Array (VLA), as part of the Disks@EVLA key program (https://safe.nrao.edu/evla/disks/; PI C. Chandler). The data are a combination $\mathrm{CnB}, \mathrm{BnA}$, and A-array data. As in the case of the SMA data, the visibilities were deprojected. The observations have a much higher signal-to-noise ratio than previous VLA observations (Hughes et al. 2007). In particular, we do not confirm the visibility null at $\sim 1000 \mathrm{k} \lambda$, which was interpreted as essential to confirm the disk structure with a 4-AU inner gap. A compact contribution from free-free emission cannot yet be excluded.

\section{Key models revisited}

As a first test, we selected 3 published models for the TW Hya disk and made a reimplemented version with a radiative-transfer code (see Sect. 4.1). These models are from Calvet et al. (2002), Ratzka et al. (2007), and Andrews et al. (2012). A full description of the model reimplementation is beyond the scope of this short paper; we refer to an 

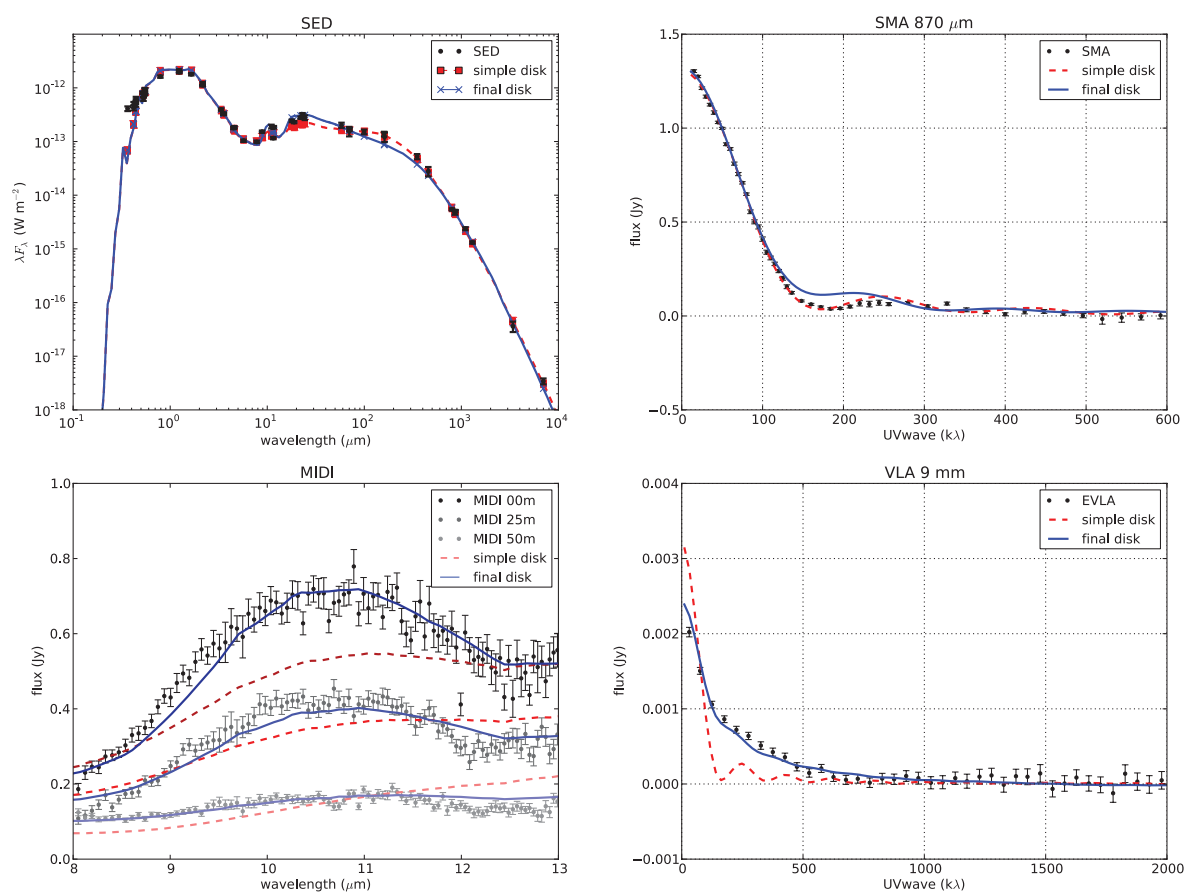

Figure 1. New disk model for the TW Hya disk (blue, full line), compared with a simple homogeneous disk model with a vertical inner rim (red, dashed line)

oncoming paper for this (Menu et al., in prep.). We implemented the complete model structure, including the dust composition and the inner-rim shape.

Apart from the fact that the models cannot simultaneously reproduce all data sets, four conclusions can be drawn:

(a) grains much larger than micron sizes are need to reproduce the SED;

(b) a full radiative transfer code is essential for modeling shadowing by the inner disk;

(c) the model SEDs all reproduce the data to some extent: degenerate SED-modeling;

(d) special care is needed in the millimeter: the new VLA data provides much stronger constraints than the old VLA data.

\section{A new model for the TW Hya disk}

\subsection{Model structure}

Since none of the three reconsidered models fully reproduces the combined data sets, we set up a new model. We use the Monte Carlo radiative-transfer code MCMax (Min et al. 2009), which allows the simulation of an azimuthally symmetric protoplanetary disk around a central star. The code fully solves the vertical hydrostatic equilibrium, and has a built-in implementation of the vertical settling of dust grains based on the $\alpha$-disk turbulence prescription (Mulders \& Dominik 2012).

For the model fitting, we use a genetic algorithm (Cantó et al. 2009). Generations of models are calculated, keeping only the best models for offspring generation. This offspring is chosen randomly in the parameter space, close to the parent parameters.

Even using intelligent fitting algorithms, there is a large interest in minimizing the number of free parameters. We kept the stellar properties fixed to literature values 
Table 1. Fitted parameters of the full-disk model shown in Fig. 1.

\begin{tabular}{lr|lr|lr}
\hline inner radius $R_{\text {in }}$ & $0.42 \mathrm{AU}$ & dust mass $<100 \mu \mathrm{m}$ & $5 \times 10^{-5} \mathrm{M}_{\odot}$ & surf. density $<100 \mu \mathrm{m} p$ & 0.7 \\
outer radius $R_{\text {out }}$ & $64 \mathrm{AU}$ & dust mass $>100 \mu \mathrm{m}$ & $6 \times 10^{-5} \mathrm{M}_{\odot}$ & surf. density $>100 \mu \mathrm{m} p_{\text {large }}$ & 1.4 \\
trans. radius $R_{\exp }$ & $3.6 \mathrm{AU}$ & width $w$ & 0.45 & turbulence parameter $\alpha$ & $10^{-5}$ \\
\hline
\end{tabular}

$\left(M_{\star}=0.6 \mathrm{M}_{\odot}, T_{\text {eff }}=4000 \mathrm{~K}, L=0.25 \mathrm{~L}_{\odot}, d=51 \mathrm{pc}\right.$; see e.g. Ratzka et al. 2007$)$. Also the dust composition was kept fixed to a mixture of amorphous olivines and carbonaceous grains ( $70 \%$ vs. $30 \%$, respectively; grain size $a=0.01-1000 \mu \mathrm{m}$, size density $\left.n(a) \propto a^{-3.5}\right)$.

\subsection{Results and discussion}

Modeling the disk with a simple disk model with a vertical inner rim ( + an empty central hole) and a homogeneous composition from small to large grains does not lead to a successful reproduction of the data, as shown in Fig. 1. We modify this simple model in two ways. First, we introduce a rounded inner rim by taking a surface density

$$
\Sigma(R)= \begin{cases}\Sigma_{\exp }\left(R / R_{\exp }\right)^{-p} \exp \left(-\left(1-R / R_{\exp }\right)^{3} / w^{3}\right) & \text { for } R_{\text {in }} \leqslant R<R_{\text {exp }}, \\ \Sigma_{\exp }\left(R / R_{\exp }\right)^{-p} & \text { for } R_{\exp } \leqslant R \leqslant R_{\text {out }}\end{cases}
$$

(Mulders et al. 2013). Here, $R_{\text {in }}, R_{\text {out }}$ and $p$ are the inner radius, outer radius, and surface density power; $\Sigma_{\text {exp }}$ is the surface density at a transition radius $R_{\exp }$, and $w$ is a dimensionless rim width. Secondly, we decouple the largest grains $(a>100 \mu \mathrm{m})$ from the smaller grains $(a<100 \mu \mathrm{m})$, and give the large grains a separate surface density power $p_{\text {large }}$ (also applying Eq. 4.1 for the surface density).

The best model obtained for this model structure is shown in Fig. 1. Although the fit to the SED and the SMA data is slightly degraded in quality, a much more accurate fit to the MIDI and VLA data is reached.

The rim shape in Eq. 4.1 follows from modeling mid-infrared interferometry of the (pre-)transitional disk object HD 100546, and is shown to be in agreement with hydrodynamical simulations of a brown dwarf opening the disk gap (Mulders et al. 2013). Our radiative transfer model shows that the MIDI data agrees with a similar inner-rim structure. Full hydrodynamical simulations of the inner-disk region would be needed to quantify the mass and orbital radius of a potential companion.

A strong concentration of larger grains towards the inner disk regions is also inferred from millimeter observations of other protoplanetary disks (e.g. Pérez et al. 2012). Rather than modeling the data at different wavelengths individually, and inferring inhomogeneous dust growth a posteriori, we assembled a model that immediately incorporates an inhomogeneous dust composition.

\section{References}

Akeson, R. L., Millan-Gabet, R., Ciardi, D. R., et al. 2011, ApJ, 728, 96

Andrews, S. M., Wilner, D. J., Hughes, A. M., et al. 2012, ApJ, 744, 162

Arnold, T. J., Eisner, J. A., Monnier, J. D., \& Tuthill, P. 2012, ApJ, 750, 119

Calvet, N., D'Alessio, P., Hartmann, L., et al. 2002, ApJ, 568, 1008

Cantó, J., Curiel, S., \& Martínez-Gómez, E. 2009, A\&A, 501, 1259

Hughes, A. M., Wilner, D. J., Calvet, N., et al. 2007, ApJ, 664, 536

Min, M., Dullemond, C. P., Dominik, C., de Koter, A., \& Hovenier, J. W. 2009, A\&A, 497, 155

Mulders, G. D. \& Dominik, C. 2012, A\& A, 539, A9

Mulders, G. D., Paardekooper, S.-J., Panić, O., et al. 2013, A\&̊A, 557, 68 
Pérez, L. M., Carpenter, J. M., Chandler, C. J., et al. 2012, ApJ (Letters), 760, L17

Quanz, S. P., Amara, A., Meyer, M. R., et al. 2013, ApJ (Letters), 766, L1

Ratzka, T., Leinert, C., Henning, T., et al. 2007, A\&A, 471, 173

\section{Discussion}

VAN DER WIEL: Are all input parameters determined by the model fitting, or are some fixed a priori? Specifically the turbulence parameter could be linked not just to how fast big grains settle, but also to spectral line shapes of molecular gas.

MENu: Even using a genetic algorithm, there is a strong interest in minimizing the number of free parameters for minimizing the computation time. Therefore, we fix parameters that can be fixed (to some extent), e.g. the stellar properties, and only let a selection of essential disk parameters vary during the fit. Concerning the turbulence parameter, it remains to be shown that the constraints we get from modeling the dust agree with observations of molecular lines. However, one needs to be careful: the assumption of constant $\alpha$-value or constant dust-to-gas ratio throughout the disk might not be entirely valid, and the disk regions dominating the $\alpha$-estimates might differ for the gas and the dust.

WEINBERGER: What do you assume about the (not well constrained) mass and temperature of the star and how does changing these affect the fit parameters?

MENu: It is indeed correct that not only the disk structure is under discussion, but also the stellar parameters. We have done an SED-fit for determining the (single-star) model that agrees best with the optical and near-infrared photometry, and take a literature value for the stellar mass $\left(0.6 \mathrm{M}_{\odot}\right)$. A few recent works have achieved better fits of the stellar spectrum with mixed spectra with different weights, which also gives clues on the stellar mass, but the uncertainties remain significant. Changing the stellar properties will alter the amount of incident radiation (for a change in luminosity) and the vertical hydrostatic equilibrium (for a change in mass), which in turn changes the temperature structure and amount of flaring. This can be expected to happen in a more or less continuous way. However, is vertical hydrostatic equilibrium a valid approximation throughout the disk? And is the stellar spectrum intercepted by the disk the same as the one we observe, given the very particular orientation of the system (pole-on) w.r.t. the observer (e.g., possible spots might be distributed very anisotropically)? Taking into account these questions might even make changes to the stellar properties irrelevant. 